\section{Delayed diagnosis of adult indolent systemic mastocytosis}

Carsten Sauer Mikkelsen, ${ }^{1}$ Andrew Nybo, ${ }^{1}$

Kristian Bakke Arvesen, ${ }^{2}$

Johan Holk-Poulsen ${ }^{1}$

'Private Practice, Broenderslev;

2Department of Dermatology, Aarhus

University Hospital, Aarhus, Denmark

\begin{abstract}
Systemic mastocytosis (SM) is a rare, heterogeneous disorder characterized by infiltration and accumulation of mast cells within multiple organs, most commonly the skin. Given the rarity of the disease and the fact that many of its symptoms are shared by more common disorders, a diagnosis may be delayed or hindered. These patients have an elevated risk of developing potentially life-threatening anaphylactoid reactions, thus underscoring the importance of keeping SM in mind as a differential diagnosis when a patient presents with chronic, itchy skin lesions and a history of multiple allergic reactions to bites, drugs, and anesthesia. We present a case illustrating that features of SM common to many disorders may hinder or delay its diagnosis.
\end{abstract}

\section{Introduction}

Systemic mastocytosis (SM) is a rare, heterogeneous disorder characterized by infiltration and accumulation of mast cells (MC) within multiple organs, most commonly the skin. ${ }^{1}$ The symptoms are shared by many other and more common disorders, which may delay or hinder the diagnosis of SM. On occasion SM can lead to life threatening anaphylactic reactions. ${ }^{2}$ For that reason SM is an import differential diagnosis in patients presenting with itchy, cutaneous lesions and a history of multiple allergic reactions or diffuse systemic symptoms.

\section{Case Report}

A 30-year-old Caucasian male with a six-toseven-year history of small, brown cutaneous lesions on his trunk and extremities was referred for evaluation.

His medical history revealed recurring itchiness and swelling of the lesions, especially after hot showers, but also from touching or rubbing on the lesions. In addition, the patient had experienced severe itchiness and flushing of the skin after an insect bite. Furthermore, the patient had experienced symptoms of mild acid reflux as well as an incidence of syncope. There was no history of either shortness of breath or bone and muscle pain.

Objective examination of the skin revealed multiple brownish, maculopapular lesions, ranging between $0.5-1.0 \mathrm{~cm}$ in diameter on the patients trunk and extremities (Figure 1). Upon rubbing on the lesions, they developed erythema, swelling and the patient complained of itchiness (positive Darier's sign).

Histological examination of a $3 \mathrm{~mm}$ skin biopsy was performed. The biopsy revealed acanthosis and intense MC infiltration of the superficial layers of the dermis, with both perivascular and diffuse infiltrates.

The patient was referred to the Department of Dermatology, Odense University Hospital for further investigation and treatment. Bone marrow analysis and a new skin biopsy was performed and they were both positive for KIT D816V-mutation.

Peripheral blood analysis was also positive for KIT D816V-mutation. In addition, an elevated level of alanine aminotransferase (ALAT) to $229 \mathrm{U} / \mathrm{L}$ (normal range, 10-70 U/L) was found. Leucocyte, hemoglobin and thrombocyte counts were normal, as were renal function (creatinine) tests and blood electrolytes (sodium and potassium). Serum tryptase level was normal, abdominal ultrasound revealed a slightly enlarged liver, consistent with steatosis, and otherwise the examination was normal. Chest X-ray examination showed no identifiable cardiothoracic or musculoskeletal abnormalities.

Finally, upper gastrointestinal endoscopy was performed, revealing hiatal hernia, gastroesophageal reflux disease, and $0.5 \mathrm{~cm}$ dark/black lesions. Multiple biopsies from the lesions were performed and histopathological examination revealed MC infiltration.

The patient was treated with clobetasolpropionat (Dermovat®) under occlusion and at discharge he was prescribed fexofenadin (Telfast@) $180 \mathrm{mg}$ two times daily, lansoprazol $30 \mathrm{mg}$ daily, and equipped with epinephrine autoinjector (EpiPen ${ }^{\circledR} \quad 0.3$ $\mathrm{mg} /$ dose) in case of an anafylactic reaction.

At follow-up three months later, the patient reported great improvement of the symptoms. Objective examination revealed remission of the skin lesions.

\section{Discussion}

We report of a patient with a six-to-sevenyear history of diffuse maculopapular lesions, intermittent itchiness and unspecific systemic symptoms. Following thorough examination, including skin biopsy with intense MC infiltra-
Correspondence: Carsten Sauer Mikkelsen, Private Practice, Bredgade 139700 Broenderslev, Denmark.

Tel. +45.201 .00198$

E-mail: c.s.mikkelsen@hotmail.com.

Key words: mastocytosis, indolent systemic masocytosis, urticaria pigmentosa, Darier's sign.

Contributions: the authors contributed equally.

Conflict of interests: the authors declare no potential conflict of interests.

Received for publication: 27 November 2013. Accepted for publication: 20 December 2013.

This work is licensed under a Creative Commons Attribution NonCommercial 3.0 License (CC BYNC 3.0).

(OCopyright C.S.Mikkelsen et al., 2014

Licensee PAGEPress, Italy

Dermatology Reports 2014; 6:5199

doi:10.4081/dr.2014.5199

tion and bone marrow analysis positive for KIT D816V-mutation, a diagnosis of indolent systemic mastocytosis was established.

$\mathrm{SM}$ is a rare, heterogeneous disease with an estimated incidence of 1 per 150.000 per year. The disease is characterized by uninhibited proliferation of MCs in the skin and/or other tissues. ${ }^{1-3}$

Mastocytosis occurs primarily in children, but there is a second peak in the incidence around age $30-50$ years. In most cases, mastocytosis in children resolves spontaneously. ${ }^{3,4}$

There are many clinical manifestations of SM and they can be attributed to both local and systemic effects of biological mediators released from MCs, as well as direct effects of infiltration of MCs in various tissues itself. Accordingly, symptoms are often divided into MC mediator derived and infiltrative. The preformed mediators including histamine, heparin, eosinophil chemotactic factors of anaphylaxis, but also enzymes like tryptase, chymase, hexosaminidase among many others, are responsible for many of symptoms of SM. ${ }^{2,5}$

Apart from IgE, possible triggers of $\mathrm{MC}$ degranulation include: venoms (insects, snakes), physical stimuli (touch, rubbing, heat and cold), chemicals (alcohol, NSAID, anesthetics, dextran, contrast media), but often the trigger for degranulation cannot be identified. $^{2,5}$ MCs are long-lived residents of vascularized tissues found in close proximity to blood vessels and nerves. As such they are capable of rapid delivery of their mediators into the circulation, which may subsequently result in widespread clinical symptoms.

A common complaint among patients with 
SM is the experience of spells, in which a sudden onset of symptoms recurs in a self-limited, stereotypic fashion. These symptoms may attribute to a wide range of causes, including cardiovascular, neurologic, physiologic, pharmacologic, and other miscellaneous causes. ${ }^{2,6}$ In SM, the sudden explosive release of MC mediators may give the outward appearance of anaphylaxis.

The most common clinical presentations of systemic mastocytosis include pruritus, flushing, urticaria pigmentosa, nausea, vomiting, diarrhea, abdominal pain, headache, palpitations, dyspnea, and syncope. ${ }^{, 7}$ As in our case, the clinical course of the disease is often characterized by a history of cutaneous lesions, which are present in up to $90 \%$ of the patients with mastocytosis, thus making the skin the most commonly involved organ. Urticaria pigmentosa is the most common cutaneous presentation in SM, seen in $56-100 \%$ of the cases. ${ }^{8}$ Solitary mastocytoma, diffuse cutaneous mastocytosis, and teleangiectasia macularis eruotiva perstans are other presentations.

A diagnosis of cutaneous mastocytosis can often be made based on the anamnesis, objective examination revealing urticaria pigmentosa, and positive Darier's sign and is confirmed by a skin biopsy with an increased number of MC.

The diagnosis of SM is based on the identification of neoplastic MCs as judged by using well-established morphological, and/or genetic
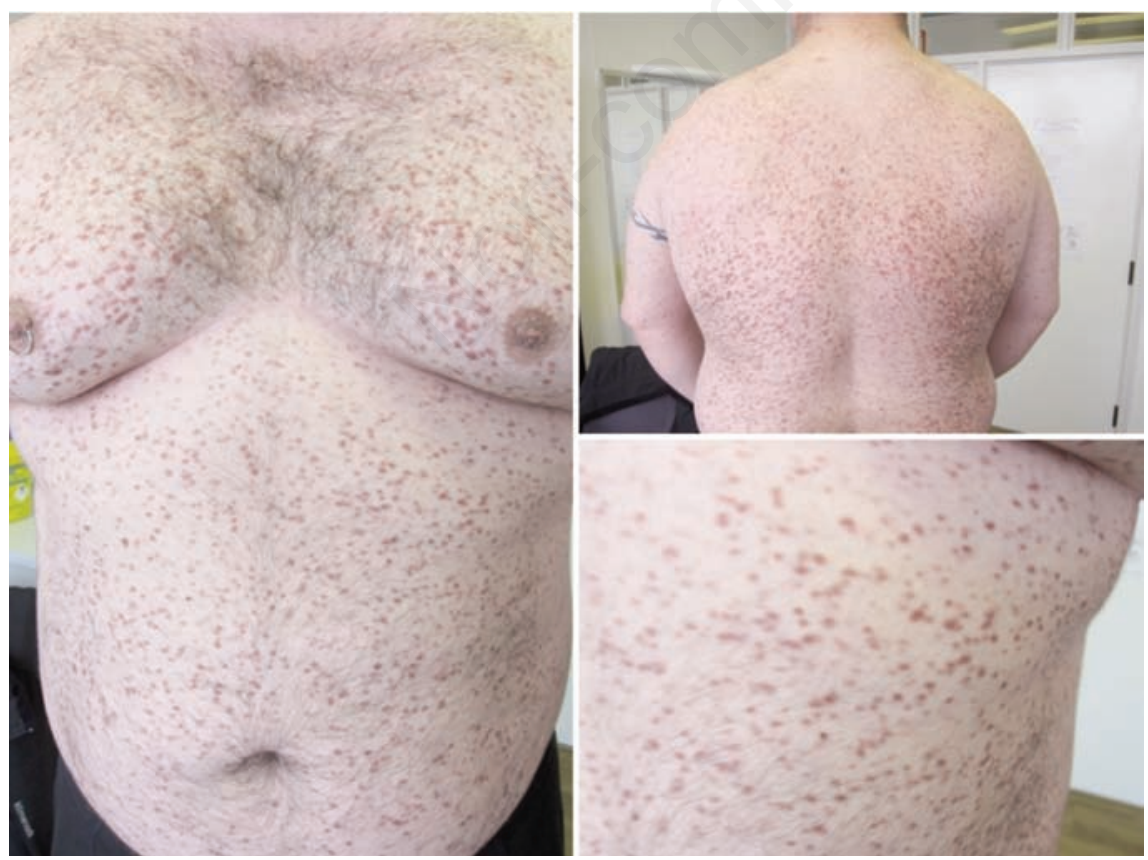

Figure 1. Maculopapular lesions on the trunk and extremities. Upon rubbing on the lesions they developed erythema, swelling and the patient complained of itchiness (positive Darier's sign). Skin revealed intense systemic mastocytosis infiltration in superficial parts of the dermis, including both perivascular and diffuse infiltrates. criteria outlined by the WHO 2008 document. $^{9}$ The diagnosis SM requires the presence of 1 major and 1 minor criterion or 3 minor criterions in the bone marrow or extracutaneous organs.

Major criterion: multifocal dense infiltrates of MCs (>15 MCs in aggregates). Minor criteria: i) MC infiltrates contain $>25 \%$ spindleshaped cells or other atypical morphologic features; ii) c-KIT D816V mutation; iii) expression of CD2 and/or CD25 on CD117+ MCs; iv) serum tryptase levels $>20 \mathrm{ng} / \mathrm{mL}$ (not valid if patient has concomitant hematologic disorder). SM includes four major subtypes: i) indolent SM (the most common of SM involving mainly the skin and bone marrow); ii) SM associated with non-mast cell clonal hematological disease (SM-AHNMD); iii) aggressive SM usually presenting without skin lesions; and iv) MC leukemia. ${ }^{1}$ Irrespective of the mastocytosis category, management of mastocytosis includes avoidance of factors triggering MC degranulation and treatment of chronic and acute mast cell mediator release. ${ }^{7,9}$ Patients, their caretakers and primary physicians should be informed of the elevated risk of anaphylactoid reactions. Treatment of such reactions include administration of intramuscular epinephrine $(0.3 \mathrm{mg})$ and $\mathrm{H}_{1}$ and $\mathrm{H}_{2}$ antihistamines. Antihistamines are also the mainstay in symptomatic treatment of pruritus, flushing and wheals. Oral cromolyn sodium has limited intestinal absorption, and is mostly used to help control gastrointestinal symptoms. Topical steroids used under occlusion may help control pruritus and decrease the number of MCs. Cytoreductive therapy is typically resolved for patients with aggressive SM and is primarily carried out in collaboration with specialists in hematology. $1,7,9,10$

\section{Conclusions}

We presented a patient with a six-to-seven year history of diffuse maculopapular lesions, intermittent itchiness and several, unspecific allergic symptoms. Given the rarity of SM and the fact the many of the symptoms are shared by more common disorders, this may hinder or delay the diagnosis. When presented with a patient with chronic, itching skin lesions and a history of multiple allergic reactions to bites, drugs, anesthesia etc. SM is an important differential diagnosis, as these patients have an elevated risk of possible life threatening anaphylactoid reactions.

\section{References}

1. Horny HP, Sotlar K, Valent P. Mastocytosis: state of the art. Pathobiology 2007;74:12132.

2. Butterfield JH. Systemic mastocytosis: clinical manifestations and differential diagnosis. Immunol Allergy Clin North Am 2006;26:487-513.

3. Almahroos M, Kurban AK. Management of mastocytosis. Clin Dermatol 2003;21:2747.

4. Ben-Amitai D, Metzker A, Cohen HA. Pediatric cutaneous mastocytosis: a review of 180 patients. Isr Med Assoc J 2005;7:320-2.

5. Robyn J, Metcalfe DD. Systemic mastocytosis. Adv Immunol 2006;89:169-243.

6. Young WF Jr, Maddox DE. Spells: in search of a cause. Mayo Clin Proc 1995;70:757-65.

7. Bains SN, Hsieh FH. Current approaches to the diagnosis and treatment of systemic mastocytosis. Ann Allergy Asthma Immunol 2010;104:1-12.

8. Brockow K. Urticaria pigmentosa. Immunol Allergy Clin North Am 2004;24: 287-316.

9. Pardanani A. Systemic mastocytosis in adults: 2011 update on diagnosis, risk stratification, and management. Am J Hematol 2011;86:362-71.

10. Pardanani A, Tefferi A. Systemic mastocytosis in adults: a review on prognosis and treatment based on 342 Mayo Clinic patients and current literature. Curr Opin Hematol 2010;17:125-32. 